\section{PTH-132 IRON DEFICIENCY ANAEMIA NURSE LED CLINIC: AUDIT OF 1ST YEAR}

doi:10.1136/gutjnl-2013-304907.619

1." C Daker, ' ${ }^{1} \mathrm{H}$ McAuley, ${ }^{1} \mathrm{I}$ Mason, 'T Shepherd, 'M Hamilton, ${ }^{1} \mathrm{O}$ Epstein, ${ }^{1} \mathrm{C}$ Murray. 'Gastroenterology, Royal Free Hospital, London, UK

Introduction Iron deficiency anaemia accounts for $4-13 \%$ of gastrointestinal referrals occurring in $2-5 \%$ of adult men and post menopausal women. Current guidelines suggest that the work up of asymptomatic anaemia remains incomplete and often inappropriate with important diagnoses of malignancy and malabsorption being missed. In an effort to improve our handling of these patients from primary care and reconfigure services, a nurse led clinic was established in 2009 with the aim to provide rapid access to appropriate investigations and management.

Methods All clinic patients between 01/06/2009-05/07/2010 were included in the study. Patients were triaged into the clinic via the GP referral letter. End points examined were:patient demographics, anaemia, microcytosis and iron deficiency, whether oral iron preparations were being used, investigations (OGD/colonoscopy), wait times for investigations and pathology identified. Data were collected using patient notes, pathology results database, clinic letters and endoscopy reports. Anaemia was classified as an $\mathrm{Hb}$ of $<11.5 \mathrm{~g} / \mathrm{dL}$.

Results 110 patients were included (no exclusions); 81 female and 29 male $(73.6 \%, 43.2 \%)$.Age range $21-90 y r s$, mean $57 y$ rs, median $58 y$ rs. $62 \%$ were made up of men (29) and post-menopausal women (47); 33\%(13) menstruating females with GI symptoms and $46 \%(21)$ menstruating females without GI symptoms.62\% were in receipt of oral iron supplementation at referral.66\% of referrals included the blood results revealing $92 \%$ anaemic; $54 \%$ microcytic; $50 \%$ iron deficient. This compared to $68 \%$ of patients anaemic bled in the clinic of whom $20 \%$ were not anaemic but on iron supplementation leaving $12 \%$ neither anaemic nor on iron supplements. Of those not on iron (42-13men, 29 women) $21 \%$ had renal disease $(\mathrm{GRF}<60), 12(29 \%)$ were iron deficient,10(24\%)were iron deficient and anaemic.A total of 61 patients underwent endoscopic investigation (47post menopausal and male group; 6 menstruating females with GI symptoms and 8 menstruating females without GI symptoms).We identified 4 mailgnancies; 3 polyps, 3 colitics, 9 upper $\mathrm{Gi}$ pathologies (GAVE, varices, ulcers, small bowel Crohns),2 with Helicobacter pylori infection and 5 with celiac disease. 12 had gynaecological causes, 2 renal causes. 46 resolved on oral iron and 8 needed parentral iron infusions. Mean time from clinic to investigation was 9.5 weeks (SD 14.8) and median 4.86 weeks.

Conclusion Of the cohort a total of $55 \%(61)$ underwent endoscopy with an overt GI pathology yield (deemed causative for the anaemia) in $26(42 \%)$. The nurse led clinic has facilitated a decreased waiting time to appropriate test so improving patient outcomes in terms patient satisfaction, time to diagnosis and management strategy implementation, decreased clinic and investigation waiting times, reduced pressure on physicians lists and most importantly morbidity and mortality.

Disclosure of Interest None Declared.

\section{PTH-133 PATIENT SATISFACTION WITH A NURSE-DELIVERED ALCOHOL LIAISON SERVICE IN A GENERAL HOSPITAL SETTING}

doi:10.1136/gutjnl-2013-304907.620

1." C Oza, ${ }^{1} \mathrm{R}$ Price, ${ }^{2} \mathrm{~S}$ Morris, ${ }^{2} \mathrm{~W}$ Lambert, ${ }^{3} \mathrm{M}$ Al-Mahbub, 'P Southern. ' $\mathrm{C}$ Gastroenterology, Bradford Royal Infirmary; ${ }^{2}$ Alcohol Care Team, Bradford District Care Trust; ${ }^{3}$ Accident \& Emergency, Bradford Royal Infirmary, Picadilly Project (Lifeline UK), Bradford, UK

Introduction NICE Guidance has mandated the need to provide an Alcohol Liaison Service (ALS) within all Acute Trusts (1). Within our organisation, we have a mature ALS which provides advice and support to prospectively identified patients admitted to the Acute Medical Service. This audit was undertaken to seek the views of service users, and to ensure that we were fulfilling their needs and expectations, thus allowing feedback on future service development.

Aim To assess the patient acceptance of a Nurse-Led Alcohol Liaison Service

Methods All patients admitted to Medical Admissions Unit (MAU), had an alcohol assessment using the validated AUDIT (2) nursing questionnaire. Those scoring $\geq 5$ were flagged to the Alcohol Liaison Service. Similarly, a high percentage of patients seen in A\&E were screened by the nurses, given extended brief advice, then offered referral to Specialist Alcohol Worker (SAW)

All patients seen by SAW, and by Alcohol Clinical Nurse Specialist (ACNS) over a 6 week period were given a questionnaire and sealed envelopes, to be returned anonymously.

Results 95 questionnaires were distributed by the ACNS, and 79 were returned (return rate 83\%). 10 questionnaires had technical printing errors, so were excluded from final analysis, so the ITT return rate was $73 \%$. 32 questionnaires were distributed by the SAW, with a $100 \%$ return rate.

The first 3 questions dealt with quality of the service, and were numerically scored from $1-4$, with 1 being poor, and 4 being excellent. When asked 'How would you rate the quality of the service you have received?' $97 \%$ answered 3 or 4 (good or excellent). When asked if the service had met their needs, $88 \%$ of clients stated that it had. The remainder of the questionnaire dealt with acceptance of the service, and whether the service would be recommended to peers. When asked whether clients would recommend a friend to the service, $90 \%$ responded positively.

The scores for the SAW were even better, with positive responses (outcome 3 or 4 on questionnaire) 100\% of the time.

Conclusion This work illustrated the acceptance and value of the ALS to service users admitted acutely. Furthermore, free text responses received were overwhelmingly supportive and positive towards the service.

Disclosure of Interest None Declared.

\section{REFERENCES}

1. Alcohol-use disorders: preventing the development of hazardous and harmful drinking, NICE Public Health Guidance 24, June 2010

2. The AUDIT (Alcohol Use Disorders Identification Test), World Health Organisation, Saunders JB, Aasland OG, Babor TF, et al. Development of the Alcohol Use Disorders Identification Test (AUDIT): WHO Collaborative Project on Early Detection of Persons with Harmful Alcohol Consumption--II. Addiction. 1993 Jun; 88(6):791-804

\section{PTH-134 TRANSNASAL ENDOSCOPY IN THE NORTHERN REGION- SURVEY TO ASSESS AVAILABILITY AND OPINION OF TNE}

doi:10.1136/gutjnl-2013-304907.621

1,"C Parker, 'S Panter. 'Gastroenterology, South Tyneside District Hospital, South Shields, UK

Introduction Transnasal endoscopy (TNE) is performed with an ultrathin endoscope via the nasal passages. It has been available for over a decade and is widely used in Japan but use is variable in Europe and there is very little data on extent of use in the UK. It is recognised to have superior patient tolerability and satisfaction and there is emerging evidence that it causes significantly less cardiovascular upset. Anecdotally we perceive this method to be rarely used in our region and we performed a survey to assess extent of its use. Methods This survey was to assess the availability of and opinion of TNE in the Northern region. The survey was circulated via email to the lead endoscopist in each of the 10 hospital trusts in the northern region. Opinion was sought on quality of views and biopsy samples and also perceived advantages and disadvantages of TNE. 
Results Of the 10 surveys sent out all recipients responded. 2 trusts have access to TNE with only one trust having access to a specific TN service performing approximately 150-200 per year. The 2 trusts with access to TNE had both received training in TNE from industry and also in-house training. The trust with a TNE service had also received training from other endoscopists experienced in TNE and an ENT surgeon. When compared with standard endoscopy $30 \%$ thought views were worse, $60 \%$ the same and $10 \%$ unable to comment (due to lack of experience of TNE). $60 \%$ thought biopsy samples were adequate, $20 \%$ too small and $20 \%$ unable to comment.

Advantages of TNE: 2 felt unable to comment due to lack of familiarity with this method. Improved patient tolerance was the main advantage stated by 7 with improved comfort, less gagging and reduced sedation requirements, with 1 stating less nursing support and therefore potential for evening lists and improving capacity issues as the main advantage.

Disadvantages: 2 unable to comment, 2 no disadvantages, 2stated cost of set up, 1- failure of nasal passage, 1-narrow channel limits therapy, 1-prolonged preparation time compared to throat spray and 1- poor views.7/8 without access to TNE felt a TNE service would be beneficial to their trust and 5 would be keen to set it up in their trust. Reported barriers to set up were cost $6 / 8$ and time 1/8. 6 would be more likely to set up a TNE service if training were available.

Conclusion TNE is not widely used in our region with only 1 of 10 trusts performing regular TNE lists. It is perceived by the majority of endoscopists to have significant patient benefit and the majority are keen to set up a service. The main restriction to use appears to be the cost of set up despite the opinion that TNE is cost efficient overall. It is indicated that making TNE training available may increase its use. This was a regional survey and it would be interesting to see if these results are replicated nationally.

Disclosure of Interest C. Parker Grant/Research Support from: Submitting author's post is funded by Imotech Medical, S. Panter: None Declared

\section{PTH-135 EXAMINING THE ATTITUDES, PERCEPTIONS AND BARRIERS OF BOWEL SCREENING WALES STAKEHOLDERS}

doi:10.1136/gutjnl-2013-304907.622

1."D Snelling, 'H Heard. 'Screening Division, Public Health UK NHS Trust, Cardiff, UK

Introduction Service evaluation is of paramount importance for the continued improvement and development of any health intervention and there is very little documented evidence that examines the attitudes and perceptions of Bowel Screeing Wales (BSW) stakeholders. Anecdotal evidence has suggested several factors that contribute towards the uptake of bowel screening in Wales, such as, lack of understanding around screening, the nature of the test, and the will to complete the test, but this evidence has not been evaluated or documented.

Methods A qualitative descriptive approach was undertaken and semi-structured interviews were conducted with stakeholders at the Royal Welsh Show, Builth Wells, Powys during July 2012 to gauge their attitudes, perceptions, and barriers towards bowel screening. Inclusion criteria was for all eligible men and women aged between 60-74 years who have been invited to be screened.

Results 42 participants agreed to take part in the interview (19 male and 23 female) of which 31 participants reported completing their bowel screening test with 12 participants reporting they had not.

The results indicate that participants are aware of cancer and have a very basic knowledge regarding bowel cancer but are not necessarily aware of the function of the bowel screening programme. A content analysis framework was developed (Newell \& Burnard, 2006) which identified two major themes; health beliefs and health behaviour. This service evaluation suggests that participant's perceived susceptibility influences their decisions to take part. Participants who are not aware of BSW or the risks associated with bowel cancer will not complete the kit. Furthermore, this service evaluation suggests that participants who do not present with symptoms are also less-likely to complete their kit. Furthermore, only a very small number of participants sited fear or anxiety as a contributing factor for participating even though they were aware that the kit was to test for cancer. Majority of the participants who declined the invitation suggested that this was due to dealing with their faecal matter. It is interesting to note that their reasons for not completing their kit were lethargy and apathy.

Conclusion Service evaluations are essential in understanding the attitudes and perceptions of stakeholders. The findings from this service evaluation suggest that participants have a limited knowledge of the risks associated with bowel cancer and know very little about the programme but perceive screening to be important. However participants perceived severity and susceptibility are contributing factors in their participation to accept or decline the invitation to be screened.

Disclosure of Interest D. Snelling Employee of: Bowel Screening Wales, H. Heard: None Declared

\section{REFERENCE}

Newell, R; Burnard, P (2006) Research for evidence-based practise. Blackwell publishing. Oxford. UK.

\section{PTH-136 FAECAL CALPROTECTIN - IS IT REQUESTED APPROPRIATELY AND IS IT COST EFFECTIVE?}

doi:10.1136/gutjnl-2013-304907.623

1, ${ }^{*}$ E Derbyshire, ${ }^{2} \mathrm{C}$ Wells, ${ }^{2} \mathrm{~J}$ Hancock, ${ }^{2} \mathrm{D}$ Dwarakanath. 'Gastroenterology, North Cumbria University Hospitals NHS Trust, Carlisle, ' Gastroenterology, North Tees \& Hartlepool NHS Foundation Trust, Stockton-on-Tees, UK

Introduction Faecal calprotectin is a neutrophil derived protein that can be detected by quantitative enzyme linked immunosorbant assay in stool samples. It reliably predicts the level of mucosal inflammation in the lower gastrointestinal tract (1). The high negative predictive value of faecal calprotectin should lead to invasive investigation being avoided with a subsequent reduction in cost and demand on already stretched endoscopy services (2). We reviewed the use of faecal calprotectin in the trust to establish if current practise confirms this.

Methods The hospital numbers of all patients who had a faecal calprotectin processed at North Tees \& Hartlepool NHS Foundation Trust from 01/04/2011 to 31/12/2011 were collected from the biochemistry department's database. These patients' case notes were then analysed to record the indication for faecal calprotectin, its result, subsequent investigation and management.

Results Faecal calprotectin was requested in 2 groups of patients: 68 with lower gastrointestinal symptoms and 44 with Inflammatory Bowel Disease (IBD). Of the 68 patients with lower gastrointestinal symptoms, faecal calprotectin was normal in 48 patients: 13 ( 9 males, 4 females, median age 30 years, commonest symptom abdominal pain) at initial presentation had no further investigation, 23 (11 males, 12 females, median age 41, commonest symptom abdominal pain) went onto have further investigations (12 colonoscopies, 3 flexible sigmoidoscopies and 1 small bowel meal and follow through; all normal); in 12 (3 males, 9 females, median age 45 , commonest symptom chronic diarrhoea) the negative faecal calprotectin was as a second line following initial investigation including 8 normal colonoscopies and 1 normal flexible sigmoidoscopy. Of those patients with IBD, faecal calprotectin was normal in 9 patients. In 7 patients management decisions were taken on the basis of its result alone. These included commencement of Infliximab $(n=2)$, 6-mercaptopurine $(n=1)$, azathoprine $(n=1)$, pentasa $(\mathrm{n}=2)$ and prednisolone $(\mathrm{n}=1)$ without further investigation. 